\title{
Explicit Formulae for 25 of the Associated Legendre Functions of the Second Kind
}

\author{
By Dietrich Suschowk
}

Since the present writer knows of no place where to find explicit expressions for some more of Legendres associated functions of the second kind than are given for instance in [1] or [2], he hopes the small collection of formulae at the end of this note might be of some use to others.

The associated Legendre functions $Q_{n}{ }^{k}(x)$ of the second kind for $|x|>1$ and nonnegative integer values of $n, k$ can be defined by [1]

$$
\begin{aligned}
Q_{n}{ }^{k}(x)=\frac{\left(x^{2}-1\right)^{k / 2}}{2^{n} n !} \frac{d^{n+k}}{d x^{n+k}}\left[\left(x^{2}-1\right)^{n} \log \frac{x+1}{x-1}\right] & \\
& -\frac{1}{2} \frac{d^{k}}{d x^{k}}\left[\log \frac{x+1}{x-1} \cdot \frac{d^{n}}{d x^{n}}\left(x^{2}-1\right)^{n}\right] .
\end{aligned}
$$

These functions can be written as follows:

$$
Q_{n}{ }^{k}(x)= \begin{cases}\frac{\left(x^{2}-1\right)^{-k / 2}}{(n-k) !}\left[\frac{1}{2} A_{n k}^{(1)}(x) \log \frac{x+1}{x-1}+A_{n k}^{(2)}(x)\right] & (n \geqq k) \\ \frac{(-1)^{k}}{\left(x^{2}-1\right)^{k / 2}} A_{n k}^{(2)}(x) & (n<k)\end{cases}
$$

where $A_{n k}^{(i)}(x)(i=1,2)$ is a polynomial and

$$
\begin{aligned}
\text { degree of } A_{n k}^{(1)}(x) & =\left\{\begin{array}{l}
n+k \text { for } n \geqq k \\
\text { no degree for } n<k\left(A_{n k}^{(1)}(x) \equiv 0\right)
\end{array}\right. \\
\text { degree of } A_{n k}^{(2)}(x) & = \begin{cases}n+k-1 \text { for } n \geqq k \\
k-n-1 \text { for } n<k\end{cases}
\end{aligned}
$$

(2) If the degree of $A_{n k}^{(i)}(x)$ is even (uneven), $A_{n k}^{(i)}(x)$ contains only even (uneven) powers of $x$.

(3) The coefficients of $A_{n k}^{(i)}(x)$ are integers.

(4) For each fixed triple $(n, k, i)$ and $n \geqq k$ the coefficients of $A_{n k}^{(i)}(x)$ have alternating signs and the coefficient of the highest power in $A_{n k}^{(1)}(x)$ is always positive and in $A_{n k}^{(2)}(x)$ always negative. For $n<k$ the coefficients of $A_{n k}^{(2)}(x)$ are all positive.

(5) If $n \geqq k>0, A_{n k}^{(1)}(x)$ has a zero of $n$th order for $x=1$.

The formulae are as follows: Let $\tau=\frac{1}{2} \log (x+1) /(x-1)$. Then

$Q_{0}{ }^{0}(x)=\tau$

$Q_{1}{ }^{0}(x)=x \tau-1$

$Q_{2}{ }^{0}(x)=\frac{1}{2}\left[\left(3 x^{2}-1\right) \tau-3 x\right]$

$Q_{3}{ }^{0}(x)=\frac{1}{3 !}\left[\left(15 x^{3}-9 x\right) \tau-15 x^{2}+4\right]$

$Q_{4}{ }^{0}(x)=\frac{1}{4 !}\left[\left(105 x^{4}-90 x^{2}+9\right) \tau-105 x^{3}+55 x\right]$ 


$$
\begin{aligned}
& Q_{0}{ }^{1}(x)=-\frac{1}{\sqrt{x^{2}-1}} \\
& Q_{1}{ }^{1}(x)=\frac{1}{\sqrt{x^{2}-1}}\left[\left(x^{2}-1\right) \tau-x\right] \\
& Q_{2}{ }^{1}(x)=\frac{1}{\sqrt{x^{2}-1}}\left[\left(3 x^{3}-3 x\right) \tau-3 x^{2}+2\right] \\
& Q_{3}{ }^{1}(x)=\frac{1}{2 \sqrt{x^{2}-1}}\left[\left(15 x^{4}-18 x^{2}+3\right) \tau-15 x^{3}+13 x\right] \\
& Q_{4}{ }^{1}(x)=\frac{1}{3 ! \sqrt{x^{2}-1}}\left[\left(105 x^{5}-150 x^{3}+45 x\right) \tau-105 x^{4}+115 x^{2}-16\right]
\end{aligned}
$$

$Q_{0}^{2}(x)=\frac{2 x}{x^{2}-1}$

$Q_{1}^{2}(x)=\frac{2}{x^{2}-1}$

$Q_{2}^{2}(x)=\frac{1}{x^{2}-1}\left[\left(3 x^{4}-6 x^{2}+3\right) \tau-3 x^{3}+5 x\right]$

$Q_{3}{ }^{2}(x)=\frac{1}{x^{2}-1}\left[\left(15 x^{5}-30 x^{3}+15 x\right) \tau-15 x^{4}+25 x^{2}-8\right]$

$Q_{4}{ }^{2}(x)=\frac{1}{2\left(x^{2}-1\right)}\left[\left(105 x^{6}-225 x^{4}+135 x^{2}-15\right) \tau-105 x^{5}+190 x^{3}-81 x\right]$

$Q_{0}^{3}(x)=-\frac{6 x^{2}+2}{\left(x^{2}-1\right)^{3 / 2}}$

$Q_{1}^{3}(x)=-\frac{8 x}{\left(x^{2}-1\right)^{3 / 2}}$

$Q_{2}{ }^{3}(x)=-\frac{8}{\left(x^{2}-1\right)^{3 / 2}}$

$Q_{3}{ }^{3}(x)=\frac{1}{\left(x^{2}-1\right)^{3 / 2}}\left[\left(15 x^{6}-45 x^{4}+45 x^{2}-15\right) \tau-15 x^{5}+40 x^{3}-33 x\right]$

$Q_{4}{ }^{3}(x)=\frac{1}{\left(x^{2}-1\right)^{3 / 2}}\left[\left(105 x^{7}-315 x^{5}+315 x^{3}-105 x\right) \tau\right.$ 
$Q_{0}^{4}(x)=\frac{24 x^{3}+24 x}{\left(x^{2}-1\right)^{2}}$

$Q_{1}^{4}(x)=\frac{40 x^{2}+8}{\left(x^{2}-1\right)^{2}}$

$Q_{2}^{4}(x)=\frac{48 x}{\left(x^{2}-1\right)^{2}}$

$Q_{3}{ }^{4}(x)=\frac{48}{\left(x^{2}-1\right)^{2}}$

$Q_{4}{ }^{4}(x)=\frac{1}{\left(x^{2}-1\right)^{2}}\left[\left(105 x^{8}-420 x^{6}+630 x^{4}-420 x^{2}+105\right) \tau\right.$

$\left.-105 x^{7}+430 x^{5}-511 x^{3}+231 x\right]$.

The above formulae have been calculated by use of the recurrence relations [2]

$$
Q_{n}^{k+2}(x)=(n-k)(n+k-1) Q_{n}{ }^{k}(x)-2(k+1) \frac{x}{\sqrt{x^{2}-1}} Q_{n}^{k+1}(x)
$$

$(n-k+1) Q_{n+1}^{k}(x)=(2 n+1) x Q_{n}{ }^{k}(x)-(n+k) Q_{n-1}^{k}(x)$

$\sqrt{x^{2}-1} Q_{n}^{k+1}(x)=(n-k) x Q_{n}{ }^{k}(x)-(n+k) Q_{n-1}^{k}(x)$

starting with

$$
Q_{0}{ }^{0}(x)=\tau, \quad Q_{0}{ }^{1}(x)=-\frac{1}{\sqrt{x^{2}-1}}, \quad Q_{1}{ }^{0}(x)=x \tau-1 .
$$

Mathematisches Institut der Technischen Hochschule, München, Germany

1. W. Magnus \& F. Oberhettinger, Formeln und Sätze für die speziellen Funktionen der Mathematischen Physik, Springer-Verlag, Berlin, 1948.

2. A. ERDELYI, Editor, Higher Transcendental Functions, vol. I, McGraw-Hill Book Co., Inc., New York, 1953. 